\title{
Original Article \\ Pattern of Antidepressant Utilization and Cost in Iran from 2006 to 2013 in Comparison with Other Countries
}

\author{
Fatemeh Soleymani ${ }^{1,2,3}$, Forouzan Taheri ${ }^{3}$, Elizabeth Roughead ${ }^{4, *}$, Shekoufeh Nikfar ${ }^{1,2}$, Mohammad Abdollahi $^{5}$ \\ ${ }^{1}$ Department of Pharmacoeconomics and Pharmaceutical Management, Faculty of Pharmacy, Tehran University of Medical Sciences, Tehran, Iran \\ ${ }^{2}$ Pharmaceutical Management \& Economics Research Center, Tehran University of Medical Sciences, Tehran, Iran \\ ${ }^{3}$ National Committee on Rational Drug Use, Food and Drug Organization, Ministry of Health and Medical Education, Tehran, Iran \\ ${ }^{4}$ Quality Use of Medicines and Pharmacy Research Centre, School of Pharmacy and Medical Sciences, University of South Australia, \\ Adelaide, South Australia 5001, Australia \\ ${ }^{5}$ Department of Toxicology and Pharmacology, Faculty of Pharmacy, Tehran University of Medical Sciences, Tehran, Iran
}

\section{ARTICLE INFO}

Article History

Received 4 March 2018

Accepted 12 September 2018

Keywords

Antidepressants

drug utilization

pharmacoepidemiology

pharmacoeconomics

\begin{abstract}
Antidepressant prescribing patterns have changed globally over the past few years, with conventional drugs including tricyclic antidepressants and monoamine oxidase inhibitors being replaced by selective serotonin reuptake inhibitors (SSRIs) and novel antidepressants. The objective of this study was to assess antidepressant utilization in Iran from 2006 to 2013 and to show Iran's situation in antidepressant consumption compared with other countries. A cross-sectional study was undertaken using prescription claims data from Iranian insurance agencies. In addition, total antidepressant sales data were obtained from the databank of the national regulatory authority. Medicines were classified according to the Anatomic Therapeutic Chemical (ATC2012 edition) System. The Organisation for Economic Co-operation and Development data were used to compare national results from Iran with other countries. Antidepressant sales were four-fold higher than those of prescribed antidepressants [24 defined daily doses (DDD)/1000 inhabitants/day were sold whereas 6 DDD/1000 inhabitants/day were prescribed in 2013]. The trend in antidepressant prescriptions and consumption showed increasing use of SSRIs (N06AB). Nortriptyline, fluoxetine, and citalopram accounted for more than $60 \%$ of all prescriptions each year. The type of adverse reactions with new expensive antidepressants may seem convincing for the growing tendency toward using these medicines, but considering their high costs, health policymakers have to be aware of the risk of overprescription of newer antidepressant. Drivers of over-the-counter purchase of antidepressants need to be explored.
\end{abstract}

(C) 2018 Atlantis Press International B.V

This is an open access article under the CC BY-NC license (http://creativecommons.org/licenses/by-nc/4.0/)

\section{INTRODUCTION}

Depression is a widespread disorder, with the World Health Organization (WHO) estimating that more than 350 million people are affected worldwide [1]. A variety of health conditions such as depressed mood, loss of interest and enjoyment, and increased fatigability may be some of the different manifestations of depression [1,2]. Depression may turn into a serious health condition, and cause poor function or even suicide, if it remains untreated [1,2].

In Iran, the prevalence of psychiatric disorders is not high compared to those recorded in international studies [3]. However, it is estimated that 7 million Iranians experience at least one psychiatric disorder, demonstrating Iran's mental health burden [4]. Based on the burden of diseases calculation, major depressive disorder is ranked as the most predominant mental health disorder in Iran [5]. Given the increasing rate of psychiatric disorders including depressive disorders, it is not surprising that the consumption of antidepressants has grown over the past decade [6-8]. The changing

\footnotetext{
*Corresponding author.Email: Libby.Roughead@unisa.edu.au
}

pattern of antidepressant use, however, is a matter of concern, because older medicines such as tricyclic antidepressants (TCAs) and monoamine oxidase inhibitors (MAOIs) have been substituted with selective serotonin reuptake inhibitors (SSRIs) and expensive newer antidepressants [9-13].

Although generic and older antidepressants have acceptable cost-effectiveness profiles [1], a greater tendency toward using newer and more expensive antidepressants has been observed among both patients and physicians. As in other countries, antidepressants are not allowed to be sold without prescription in Iran, but owing to the lack of monitoring and enforcement, these medicines are available in pharmacies illegally without prescription. This may increase the risk of unnecessary antidepressant use, and considering the high tendency toward use of expensive medicines, this will make it inevitable for health policymakers to put more emphasis on understanding the changes in prescribing patterns and the escalating trend of antidepressant costs. Although much is known about antidepressant use in high-income countries, information on antidepressant use in Middle Eastern countries including Iran is lacking. This study aimed to assess national trends in antidepressant utilization for Iran. 


\section{MATERIALS AND METHODS}

A cross-sectional design was used to identify the pattern of antidepressant utilization in the Iranian population from 2006 to 2013. The study protocol is registered with the Iran Food and Drug Administration (IFDA) (No. 658/90216) and this registration number permits access to use the national data.

Prescription claims data were obtained from the Iranian insurance agencies during the 7 years of the study. There are two main insurance agencies in Iran, both of which are government insurance companies. These insurance agencies cover more than $70 \%$ of the national prescriptions, and the data regarding all prescriptions are recorded there. We included all prescriptions with at least one antidepressant in this study.

Sales data from the databank of the national regulatory authority contain data on all medicines sold by wholesalers to the pharmacies. The difference between total sales and the prescription claims data indicates the amount for which there is no valid prescription.

Prescriptions containing at least one dosage form of antidepressant medications were screened. The antidepressants listed in the national formulary were classified according to the Anatomic Therapeutic Chemical (ATC-2012 edition) System [14]. Drug name, strength, form, quantity, price, and date of supply were extracted from prescription claims. Additionally, the same data related to the sales of antidepressant agents in Iran were obtained from the official data bank of the national regulatory authority.

Using the WHO-defined daily doses (DDD) for medicines [14], the DDD/1000 inhabitants/day were calculated for each year using prescription claims data and sales data as well as national population data during the study period. The formula used was:

$$
\mathrm{DDD} / 1000 \text { per day }=\frac{\begin{array}{l}
\text { Mass }(\text { strength }) \times \text { Quantity } \\
\times \text { Number of dispensings }(\text { packs }) \times 1000
\end{array}}{\text { DDD } \times \text { Population } \times \text { Number of days }}
$$

where Mass is the mass or strength of the product, Quantity is the quantity in the pack supplied, and DDD is the defined daily dose. The population refers to the population that is relevant to the data that have been used. Number of days is the count of days in the time period (i.e., 365 for 1 year) [14].

The Organisation for Economic Co-operation and Development (OECD) health statistics data on pharmaceutical consumption were extracted from the OECD website to show where Iran stands among other countries [15].

Population statistics were obtained from the World Bank data [16]. Antidepressant use was also reported by annual frequencies (and percentages) and costs.

\section{RESULTS}

More than 600 million prescriptions were reviewed. The number of DDD/1000 inhabitants/day of antidepressant medications obtained on prescription was 3 in 2006 and rose to 6 in 2013. When examining sales data, which include both prescriptions and self-medication (bought without prescription), the antidepressant medication sales rose from 16 DDD/1000 inhabitants/day in 2006 to 24 DDD/1000 inhabitants/day in 2013. A considerable difference between the amount of antidepressant prescription data and sales was observed, highlighting that overthe-counter purchases were greater than prescribed ones (see Fig. 1).

The trend in antidepressant medications class by prescription sales and over-the-counter sales is depicted by Figs. 2 and 3. The largest growth in prescribed antidepressants was with SSRIs (N06AB), which increased from 0.97 in 2006 to 3.45 in 2013 (results are all given in DDD/1000 inhabitants/day). At the same time, the amount of prescribed nonselective monoamine reuptake inhibitors (N06AA) increased slightly from 0.96 DDD/1000 inhabitants/ day in 2006 to $1.63 \mathrm{DDD} / 1000$ inhabitants/day in 2013. In terms of individual medicines, nortriptyline was the medicine with the largest prescription volume, followed by fluoxetine and citalopram.

The total price of antidepressant medications sold from wholesale to retail pharmacies and the percentage of the relevant cost for each of these medications is summarized in Table 1.

The number of prescribed antidepressant medications and the percentage of the relevant amount of prescribed antidepressants are summarized in Table 2.

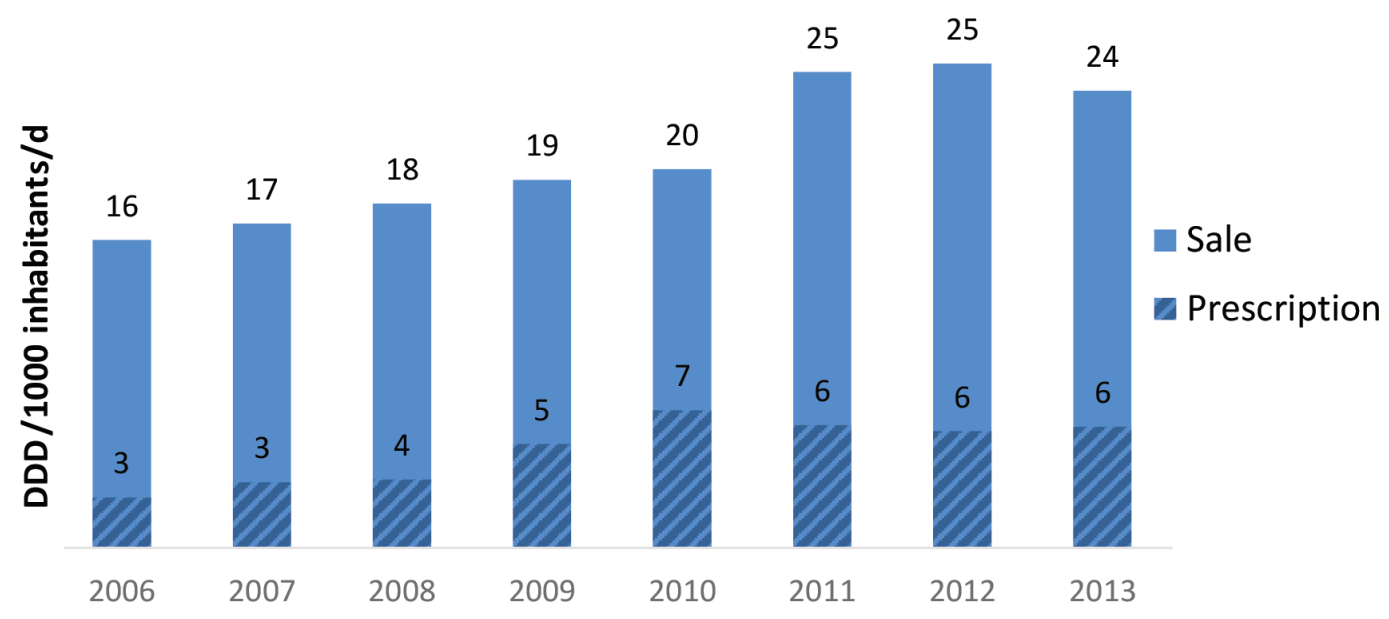

Figure $1 \mid$ Defined daily doses (DDD)/1000 inhabitants/day (sales) in comparison to DDDs/1000 inhabitants/day of prescribed antidepressants (2006-2013) 


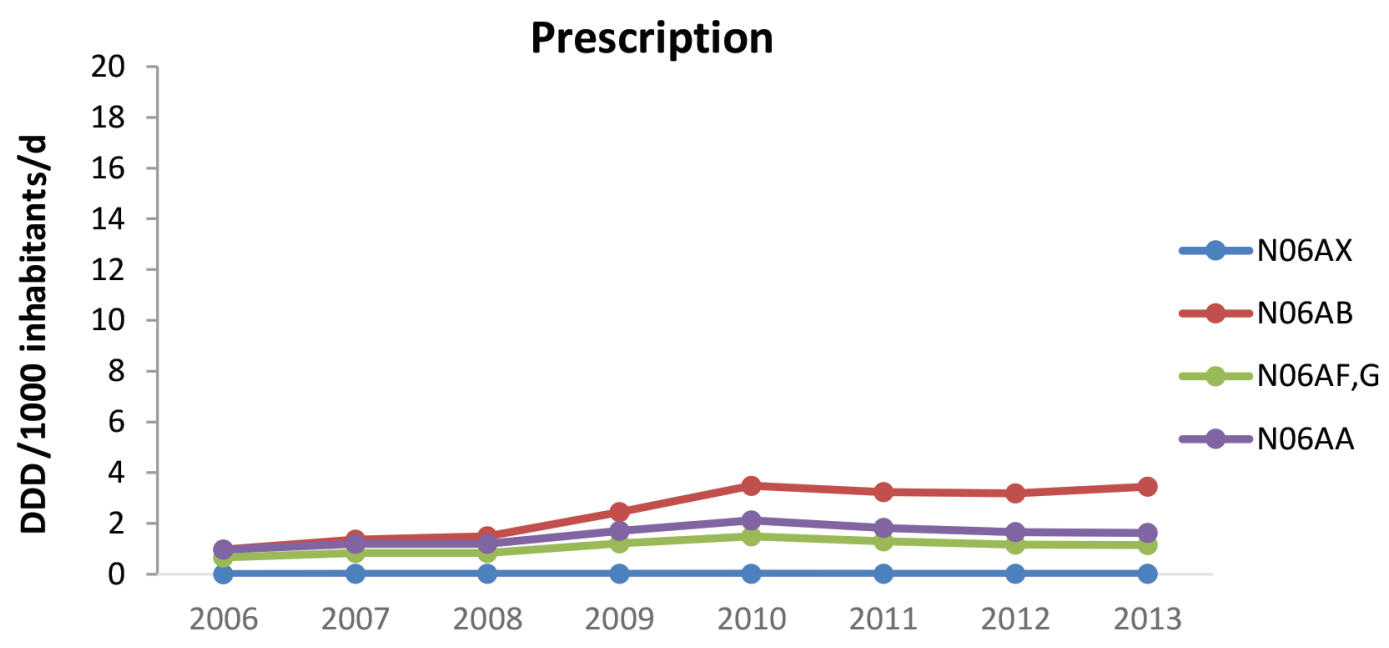

\begin{tabular}{|l|l|l|l|}
\hline N06 & Nonselective monoamine & N06A & Monoamine oxidase \\
AA & reuptake inhibitors & F,G & inhibitors \\
\hline N06 & Selective serotonin reuptake & N06A & Other \\
AB & inhibitors & X & antidepressants \\
\hline
\end{tabular}

Figure 2 Antidepressants prescription trend (DDD/1000 inhabitants/day, 2006-2013)

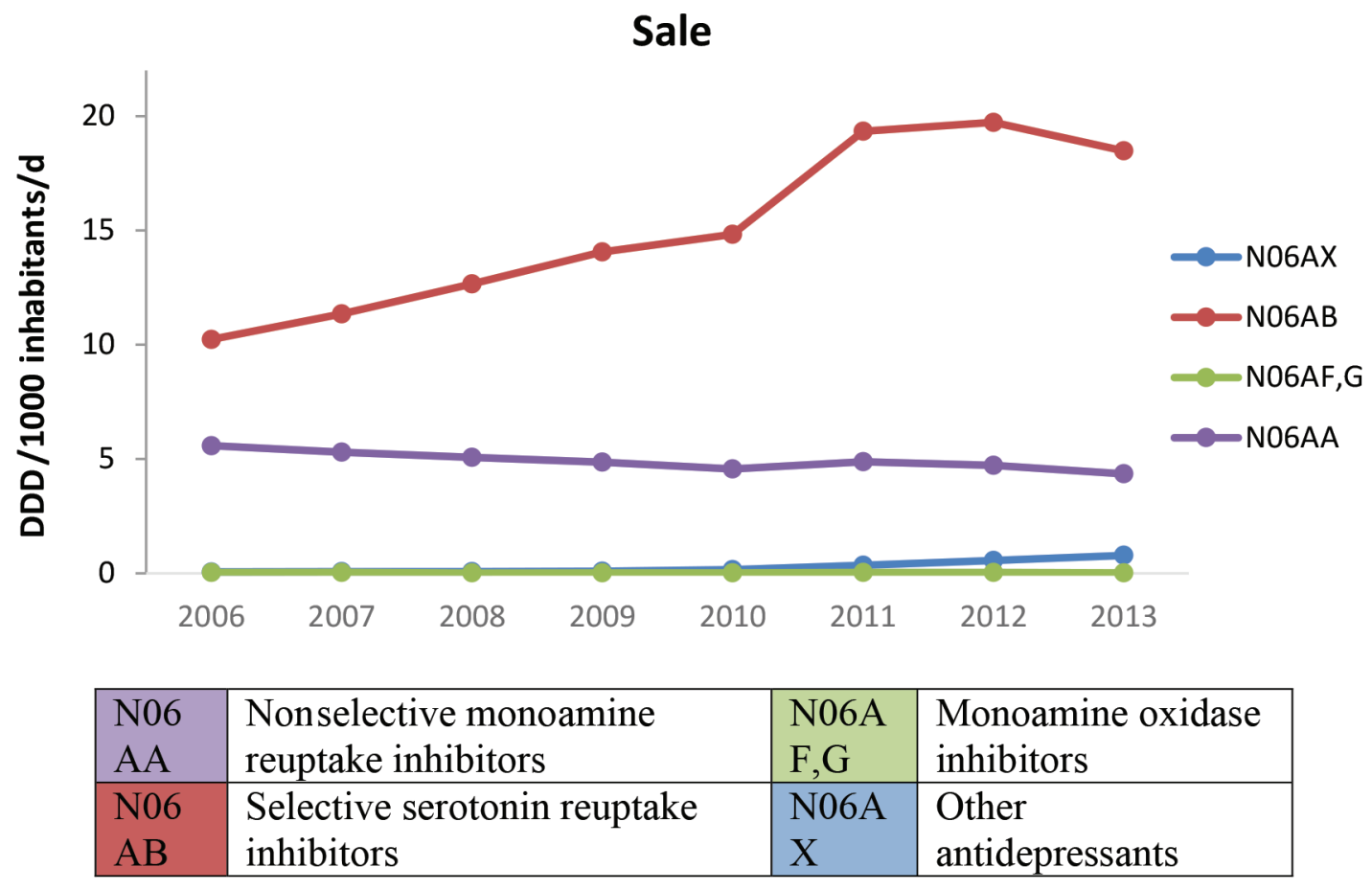

Figure 3 Antidepressants sales trend (DDD/1000 inhabitants/day, 2006-2013)

\section{DISCUSSION}

Although there are several studies from Iran assessing the burden of psychiatric disorders [3-5], specific data about the prescribing pattern of different therapeutic classes of antidepressant agents are lacking.
The results of the present study show that overall consumption of antidepressants in Iran has risen 50\% in an 8-year period. This ascending trend is consistent with rising use in Asian, European, American, and other countries, including Australia and UK [8,17-20]. 

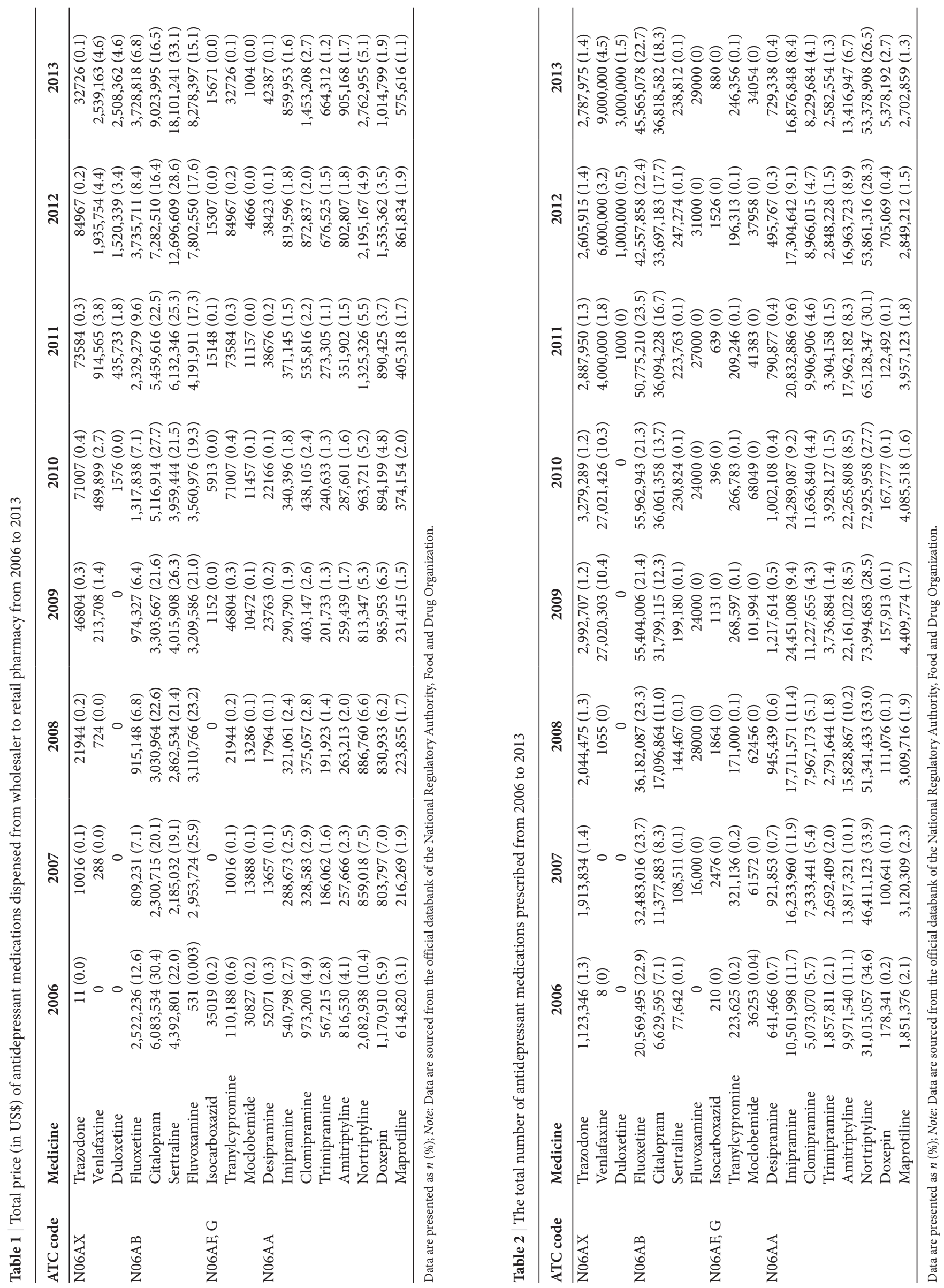
Evaluation of the consumption and prescription pattern of different categories of antidepressant agents in Iran showed that the amount of sold nonselective monoamine reuptake inhibitors (N06AA) decreased, whereas the amount of SSRI (N06AB) medications that were prescribed and sold increased throughout the study period.

The literature illustrates a wide variation in antidepressant use between different countries. A 6-month (from May 2004 to September 2005) observational, prospective, multicenter study conducted in 12 European countries (Austria, Belgium, France, Germany, Ireland, Italy, The Netherlands, Norway, Portugal, Sweden, Switzerland, and the UK) showed that although there was a wide variation between countries, overall prescriptions of SSRIs were significantly higher in comparison with those of other antidepressants (63.3\%) [17].

Based on the results of an international study of antidepressant prescribing patterns in 20 hospitals in East Asia from 1898 patients (October 2003-March 2004), among newer antidepressants, SSRIs and other newer antidepressants were prescribed for $50.4 \%$ and $26.6 \%$ of patients, respectively. The highest number of prescribed medicines was paroxetine 426 (22/4\%), fluoxetine $264(13 / 8 \%)$, trazodone 226 (11/9\%), fluvoxamine $159(8 / 4 \%)$, sertraline $128(6 / 7 \%)$, citalopram $125(6 / 6 \%)$, amitriptyline 119 $(6 / 3 \%)$, and mirtazapine [8].

Another study, which was carried out in Australia from 2000 to 2011, showed that sertraline, escitalopram, and venlafaxine were the most dispensed antidepressants [18] (Fig. 4).

Antidepressant prescribing patterns appear to vary to a great extent depending on the country from which the prescriptions are collected-for example, high use of TCAs in Germany has been indicated in three studies [17,21,22], whereas SSRIs and other newer antidepressants are more commonly used in other European and East Asian countries and Australia. One possible explanation for these differences would be the variety of national formularies, which results in variations in the availability of antidepressants [17].

In this study, although the trend for TCA consumption was falling throughout the study period, sales and administration of some TCAs (N06AA) such as nortriptyline remained high. The total cost of TCAs medicines was lower than that of other antidepressants such as SSRIs and newer antidepressants. The newer generation of antidepressants accounted for more than $75 \%$ of total antidepressants' cost nationwide, which is attributable to the high cost of these medicines compared with older antidepressants.

The increasing rate of antidepressant consumption may be a result of better methods of recognizing mental health problems and increasing social awareness regarding mental disorders [18]. Considering the almost equal effectiveness of TCAs and SSRIs and newer antidepressants and the high cost of new antidepressants, the only explanation for the growing tendency toward prescribing and consuming new and expensive generation of antidepressants may be the perceived differences in efficacy or side effects, their safety in overdose, and their longer half-lives with a single daily dose regimen $[15,17]$. All of these benefits, however, should be compared to the high cost of these medicines.

Economic and cultural factors such as previous use of medication, ease of access to nonprescribed medication, availability of drugs, and other people's recommendations, result in widespread antidepressant self-medication [23]. A study showed that psychiatric conditions are among the most common self-medicated diseases

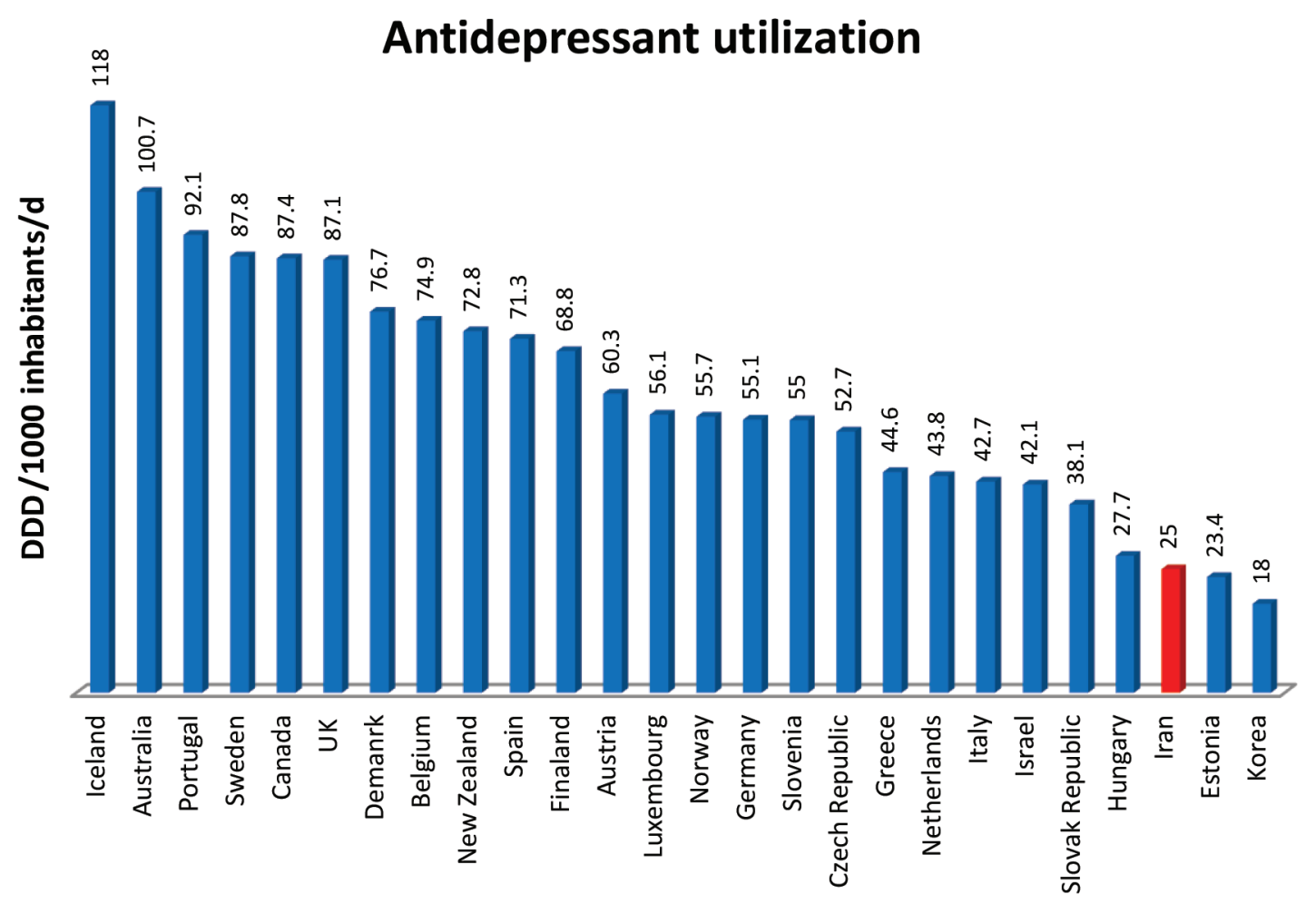

Figure 4 Antidepressant utilization: selected countries, 2012 (source: OECD Health Statistics and Ministry of Health, Iran) [15] 
in Iran [23]. Therefore, it is not surprising that the amount of prescribed antidepressants is significantly lower than the total consumption of these medicines.

The accuracy of self-diagnosis-and thus the appropriateness of medicine use-is unclear where self-medication occurs. Inappropriate medicine use may lead to possible undesirable effects. Thus, in addition to the high cost of newer antidepressants, which are in many cases used by patients without prescription, undesired effects of these medicines may cause unnecessary costs to Iran's health system.

\subsection{Study Limitation}

The prescription claims data from Iranian insurance agencies and National Committee of Rational Use of Drug (NCRUD) prescribing database does not contain information on all prescriptions. These data have been extracted from the Health Insurance Organization claims database [24]. Furthermore, the National Committee of Rational Use of Drug prescribing database does not contain information on diagnosis. In addition, patients' demographic information was unavailable; thus, we could not determine the appropriateness of the antidepressant therapy.

A strength of this study was the ability to triangulate data from two sources: the health insurance claims databases and the sales database. These two datasets combined provided stronger evidence of the increasing trend and showed the large rate of over-the-counter, potential self-medication use ( $75 \%$ of use in 2013$)$.

\section{CONCLUSION}

Selective serotonin reuptake inhibitors and new-generation antidepressants are used increasingly in Iran. These medicines are considerably more expensive than TCAs and MAOIs with different side effects and similar effectiveness. We found much higher rates of antidepressant use when analyzing sales data compared to prescription data. This difference between prescription amount of antidepressants and sales data suggests significant self-medication. Monitoring of the prescription and consumption pattern as well as investigating the pharmacoeconomics factors and drivers of overthe-counter use may help policymakers to improve monitoring systems and improve antidepressant use.

\section{AUTHORS' CONTRIBUTIONS}

FS designed the study with contributions from FT and SN. FS and FT collected data. FS analyzed the data and was responsible for data validation. FS, FT, and SN wrote the initial draft. FS, MA, and ER refined successive drafts. All authors approved the final version of the manuscript. ER is the guarantor.

\section{CONFLICTS OF INTEREST}

The authors declare no conflicts of interest.

\section{REFERENCES}

[1] Marcus M, Yasamy MT, van Ommeren M, Chisholm D, Saxena S. Depression. A global public health concern. Depression: A Global Crisis. World Federation for Mental Health, Vol. 1, Occoquan, VA; 2012; p. 6-8.

[2] Duthey B. Priority medicines for Europe and the world: "a public health approach to innovation". Geneva: WHO Background Paper, 2013; p. 6.

[3] Noorbala AA, Bagheri Yazdi SA, Yasamy MT, Mohammad K. Mental health survey of the adult population in Iran. Br J Psychiatry 2004;184;70-3.

[4] Mohammadi MR, Davidian H, Noorbala AA, Malekafzali H, Naghavi HR, Pouretemad HR, et al. An epidemiological survey of psychiatric disorders in Iran. Clin Pract Epidemiol Ment Health 2005; $1 ; 16$.

[5] Naghavi M, Abolhassani F, Pourmalek F, Lakeh MM, Jafari N, Vaseghi S, et al. The burden of disease and injury in Iran 2003. Popul Health Metr 2009;7;9.

[6] Olfson M, Marcus SC, Pincus HA, Zito JM, Thompson JW, Zarin DA. Antidepressant prescribing practices of outpatient psychiatrists. Arch Gen Psychiatry 1998;55;310-16.

[7] Pratt LA, Brody DJ, Gu Q. Antidepressant use in persons aged 12 and over: United States, 2005-2008. NCHS Data Brief, 2011;76;1-8.

[8] Uchida N, Chong MY, Tan CH, Nagai H, Tanaka M, Lee MS, et al. International study on antidepressant prescription pattern at 20 teaching hospitals and major psychiatric institutions in East Asia: Analysis of 1898 cases from China, Japan, Korea, Singapore and Taiwan. Psychiatry Clin Neurosci 2007;61;522-8.

[9] Guaiana G, Andretta M, Corbari L, Mirandola M, Sorio A, D’Avanzo B, et al. Antidepressant drug consumption and public health indicators in Italy, 1955 to 2000. J Clin Psychiatry 2005;66;750-5.

[10] Lawrenson RA, Tyrer F, Newson RB, Farmer RD. The treatment of depression in UK general practice: selective serotonin reuptake inhibitors and tricyclic antidepressants compared. J Affect Disord 2000;59;149-57.

[11] Martín-Agueda B, López-Muñoz F, Rubio G, Guerra JA, Silva A, Álamo C. Management of depression in primary care: a survey of general practitioners in Spain. Gen Hosp Psychiatry 2005;27;305-12.

[12] Olié JP, Elomari F, Spadone C, Lépine JP. [Antidepressants consumption in the global population in France]. Encephale 2002;28;411-17 (in French).

[13] van Marwijk HW, Bijl D, Adegrave HJ, de Haan M. Antidepressant prescription for depression in general practice in The Netherlands. Pharm World Sci 2001;23:46-9.

[14] World Health Organisation (WHO). WHO Collaborating Center for Drug Statistics Methodology. ATC/DDD Index 2018. Available from: https://www.whocc.no/atc_ddd_index/ (accessed December 5, 2018).

[15] Organisation for Economic Co-operation and Development (OECD). Health policies and data. Health at a Glance: Europe 2014. Available from: https://www.oecd.org/els/health-systems/ Health-at-a-Glance-Europe-2014-CHARTSET.pdf (accessed December 5, 2018).

[16] The World Bank. Population, total. Available from: https://data. worldbank.org/indicator/SP.POP.TOTL?locations=IR (accessed December 5, 2018). 
[17] Bauer M, Monz BU, Montejo AL, Quail D, Dantchev N, Demyttenaere K, et al. Prescribing patterns of antidepressants in Europe: results from the Factors Influencing Depression Endpoints Research (FINDER) study. Eur Psychiatry $2008 ; 23 ; 66-73$.

[18] Stephenson CP, Karanges E, McGregor IS. Trends in the utilisation of psychotropic medications in Australia from 2000 to 2011. Aust N Z J Psychiatry 2013;47;74-87.

[19] Marcus SC, Olfson M. National trends in the treatment for depression from 1998 to 2007. Arch Gen Psychiatry 2010;67;1265-73.

[20] Ilyas S, Moncrieff J. Trends in prescriptions and costs of drugs for mental disorders in England, 1998-2010. Br J Psychiatry 2012;200;393-8.
[21] Ufer M, Meyer SA, Junge O, Selke G, Volz HP, Hedderich J, et al. Patterns and prevalence of antidepressant drug use in the German state of Baden-Wuerttemberg: a prescription-based analysis. Pharmacoepidemiol Drug Saf 2007;16;1153-60.

[22] Fegert JM, Kölch M, Zito JM, Glaeske G, Janhsen K. Antidepressant use in children and adolescents in Germany. J Child Adolesc Psychopharmacol 2006;16;197-206.

[23] Azami-Aghdash S, Mohseni M, Etemadi M, Royani S, Moosavi A, Nakhaee M. Prevalence and cause of self-medication in Iran: a systematic review and meta-analysis article. Iran J Public Health 2015;44;1580-93.

[24] Soleymani F, Valadkhani M, Dinarvand R. Challenges and achievements of promoting rational use of drugs in Iran. Iran J Public Health 2009;38;166-8. 